\title{
Interferon-alpha or -beta facilitates SARS-CoV-2 pulmonary vascular infection by inducing ACE2
}

\author{
Timothy Klouda ${ }^{1}$ Yuan Hao ${ }^{1}$. Hyunbum Kim ${ }^{1}$. Jiwon Kim ${ }^{1}$ - Judith Olejnik ${ }^{2,3}$ - Adam J. Hume ${ }^{2,3}$. \\ Sowntharya Ayyappan ${ }^{1} \cdot$ Xuechong Hong ${ }^{4} \cdot$ Juan Melero-Martin ${ }^{4} \cdot$ Yinshan Fang $^{5}$. Qiong Wang ${ }^{6} \cdot$ Xiaobo Zhou $^{7}$. \\ Elke Mühlberger ${ }^{2,3} \cdot$ Hongpeng Jia $^{6} \cdot$ Robert F. Padera Jr. $^{8} \cdot$ Benjamin A. Raby ${ }^{1} \cdot$ Ke Yuan $^{1}{ }^{10}$
}

Received: 7 September 2021 / Accepted: 20 October 2021 / Published online: 29 October 2021

(c) The Author(s) 2021

\begin{abstract}
Severe viral pneumonia caused by the severe acute respiratory syndrome coronavirus 2 (SARS-CoV-2) is characterized by a hyperinflammatory state typified by elevated circulating pro-inflammatory cytokines, frequently leading to potentially lethal vascular complications including thromboembolism, disseminated intracellular coagulopathy and vasculitis. Though endothelial infection and subsequent endothelial damage have been described in patients with fatal COVID-19, the mechanism by which this occurs remains elusive, particularly given that, under naïve conditions, pulmonary endothelial cells demonstrate minimal cell surface expression of the SARS-CoV-2 binding receptor ACE2. Herein we describe SARS-CoV-2 infection of the pulmonary endothelium in postmortem lung samples from individuals who died of COVID-19, demonstrating both heterogeneous ACE2 expression and endothelial damage. In primary endothelial cell cultures, we show that SARS-CoV-2 infection is dependent on the induction of ACE2 protein expression and that this process is facilitated by type 1 interferon-alpha (IFN $\alpha$ ) or -beta( $\beta$ ) - two of the main anti-viral cytokines induced in severe SARS-CoV-2 infection-but not significantly by other cytokines (including interleukin 6 and interferon $\gamma / \lambda$ ). Our findings suggest that the stereotypical anti-viral interferon response may paradoxically facilitate the propagation of COVID-19 from the respiratory epithelium to the vasculature, raising concerns regarding the use of exogenous IFN $\alpha / \beta$ in the treatment of patients with COVID-19.
\end{abstract}

Keywords Endothelial $\cdot$ ACE2 $\cdot$ Interferon $\cdot$ SARS-CoV-2 $\cdot$ COVID-19

Timothy Klouda and Yuan Hao have contributed equally to the work.

Ke Yuan

Ke.Yuan@childrens.harvard.edu

1 Division of Pulmonary Medicine, Boston Children's Hospital and Harvard Medical School, Boston, MA 02115, USA

2 Department of Microbiology, Boston University School of Medicine, Boston, MA 02118, USA

3 National Emerging Infectious Diseases Laboratories, Boston University, Boston, MA 02118, USA

4 Department of Cardiac Surgery, Boston Children's Hospital and Harvard Medical School, Boston, MA 02115, USA

\section{Introduction}

More than 244 million severe acute respiratory syndrome coronavirus 2 (SARS-CoV-2) infections have been confirmed in the one year since its identification. Though the majority of patients survive, with disease limited to mildto-moderate respiratory involvement, the virus has already

5 Center for Human Development and Division of Digestive and Liver Disease, Department of Medicine, Columbia University Medical Center, New York, NY 10032, USA

6 Division of Pediatric Surgery, Department of Surgery, The Johns Hopkins University School of Medicine, Baltimore, MD 21205, USA

7 Division of Pulmonary and Critical Care Medicine, Channing Division of Network Medicine, Brigham and Women's Hospital, Harvard Medical School, Boston, MA 02115, USA

8 Department of Pathology, Brigham and Women's Hospital, Harvard Medical School, Boston, MA 02115, USA 
claimed more than 4 million lives, largely a consequence of severe pneumonia, acute respiratory distress syndrome (ARDS) and respiratory failure [1]. Though diffuse alveolar damage is a nearly ubiquitous finding in these cases, physiologic evidence of pulmonary vascular dysfunction is frequently observed, and a subset of patients (particularly those with hypertension, diabetes or obesity) develop extrapulmonary vascular complication - thrombosis, thromboembolism, coagulopathy - with a five to tenfold higher mortality rate [2-6]. Autopsy studies have reported widespread thrombosis with microangiopathy and alveolar capillary microthrombi throughout the pulmonary vasculature [7]. In addition, the frequent observations in severe COVID-19 of marked elevations of plasma von Willebrand Factor (vWF), P-selectin, D-dimers and other coagulation activating factors provide indirect evidence that endothelial damage and the development of vasculopathy as a pivotal determinant of clinical outcome in many patients.

Our study identified the co-presence of SARS-CoV-2 nucleoprotein $(\mathrm{N})$ and Angiotensin-converting enzyme 2 (ACE2) receptor on the pulmonary vascular endothelium in postmortem COVID-19 patient samples. Moreover, the vascular distribution of endothelial viral infection (positive anti-SARS-CoV-2 N staining) and disrupted endothelial structure were observed mostly in larger muscularized arteries and veins (diameter $>100 \mu \mathrm{m}$ ) in four out of six samples. Importantly, we also demonstrated that pro-inflammatory IFN $\alpha$ or $\beta$ induced ACE 2 on primary human pulmonary endothelial cell cultures and showed for the first time that primary endothelial cells were successfully infected with wild type SARS-CoV-2 or transduced by SARS-CoV-2 spike protein pseudotyped HIV viruses. In addition, our study suggested that IFN $\alpha$ or $\beta$ induced functional ACE2, rather than the proposed non-functional delta (d) ACE2. Our work proposed the critical role of ACE2 upregulation on pulmonary endothelial cells by IFN $\alpha$ or $\beta$ contributed to the understanding of COVID-19 associated vasculopathy, aiding future research on characterizing SARS-CoV-2 infection in cardiovascular biology. Translationally, our findings also raised concerns about using exogenous IFN $\alpha$ or $\beta$ treaments in patients with severe COVID-19.

\section{Results}

\section{Viral staining was positive mostly in larger muscularized arteries and veins}

To evaluate the role of the pulmonary endothelium in this process, we applied super high-resolution microscopy and immunofluorescence (IF) staining to post-mortem lung samples from six adults (patient \#1-\#6) who died of complications of COVID-19. Consistent with the epidemiology of severe COVID-19, these individuals were older (ages 53-76), and all but one (patient \#3) were hypertensive and/ or diabetic and had histories of significant comorbidities (Table 1). Acute respiratory failure was a proximate cause of death in all six cases, and five of six cases had histopathologic evidence of diffuse alveolar damage at autopsy (Fig. 1, patient \#3 as a representative). We assessed the vascular distribution of SARS-CoV-2 infection by IF staining of paraffin embedded lung samples with antibodies recognizing the SARS-CoV-2 N protein (anti-SARS-CoV-2 N) and the endothelial surface marker CD31 (anti-CD31) (Fig. 1). Though SARS-CoV-2 N staining was most predominantly distributed in Type I/II pneumocytes lining alveolar sacs and sloughed cellular debris in the airspaces (Fig. 1B inside air sacs with a bright red color), co-localization of anti-SARSCoV-2 N and anti-CD31 antibodies consistent with endothelial SARS-CoV-2 infection was observed in samples from 4 of 6 subjects (Patient \#1-\#4, Fig. 1B; Suppl Figs. 1, 2, 3). The vascular distribution of endothelial viral infection was not uniform, observed mostly in larger (diameter $>100 \mu \mathrm{m}$ ) muscularized arteries (Fig. 2, 2nd and 3rd rows) and veins (Suppl Fig. 2, 3rd row) but absent in arterioles and vessels smaller than $50 \mu \mathrm{m}$ in diameter (Fig. 2; Suppl Figs. 1, 2, $3,4,5$, all 1 st rows), including those in proximity to infection in adjacent pneumocytes. In these larger vessels, the presence of viral infection was consistently accompanied by signs of endothelial damage and vasculopathy, including disruption of the endothelial lining, sloughing of endothelial cells (ECs) into the vascular lumen, and apoptotic ECs with distorted nuclear shape (Fig. 2; Suppl Figs. 1, 2, 3, arrowheads). In the inner layer of blood vessels, we found the alignment of CD31 positive cells were discontinuous and most of them were sloughing off, thus the integrity of endothelial layers seen in patients \#1-\#4 was severely disrupted, whereas patients \#5 and \#6 demonstrated well preserved endothelial integrity and no obvious disruption. Additionally, no obvious vasculopathy was found in these two patients without evidence of SARS-CoV-2 endothelial infection (Suppl Figs. 4, 5), nor in the smaller vessels in patients (\#1-4) with large-vessel endotheliitis, suggesting direct viral cytopathic effects as an inciting cause of damage. Though there were no obvious clinical differences between those with and without endotheliitis, histologic evidence of microvascular thrombosis was limited to those with endothelial damage in 3 of 4 subjects (Patient \#1, 2, 3), including one subject (\#3, Fig. 2) who was also found on post-mortem gross inspection to have a subsegmental pulmonary embolism. No evidence of thrombosis was observed in the two subjects (Patient \#5, \#6) who did not have SARS-CoV-2 vascular infection. Together, these findings suggested direct pulmonary endothelial infection with resultant endothelial damage as proximal events in the promotion of vascular thrombosis in COVID-19. 
Table 1 Patient characteristics

Patients with evidence of endothelial infection and damage

Patient 1

\section{Demographics}

Age (Years)

Gender

Race

Smoking status

Co-morbidities

BMI $(\mathrm{kg} / \mathrm{m} 2)$

Hypertension

Diabetes

Other medical conditions

\begin{tabular}{|c|c|}
\hline $\begin{array}{l}\text { Autoimmune/ } \\
\text { inflammatory }\end{array}$ & $\begin{array}{l}\text { SLE, RA, ILD / PF, } \\
\text { CKD, MGUS }\end{array}$ \\
\hline $\begin{array}{l}\text { Cardiopulmonary/ } \\
\text { vascular }\end{array}$ & CAD \\
\hline \multicolumn{2}{|l|}{ Medications } \\
\hline Immunosuppression & $\begin{array}{l}\text { Prednisone, Tofaci- } \\
\text { tinib }\end{array}$ \\
\hline $\begin{array}{l}\text { RAAS interacting } \\
\text { drug }\end{array}$ & None \\
\hline \multicolumn{2}{|c|}{ COVID-19 course and management } \\
\hline \multicolumn{2}{|l|}{ Radiologic findings } \\
\hline Ground glass (CT) & Bilateral \\
\hline $\begin{array}{l}\text { Consolidation (CT } \\
\text { or CXR) }\end{array}$ & Yes \\
\hline $\begin{array}{l}\text { Mechanical ventila- } \\
\text { tion }\end{array}$ & Yes \\
\hline Ventilation mode & $\mathrm{VC}$ \\
\hline PEEP(cmH2O) & 14 \\
\hline $\mathrm{FiO} 2$ & 0.4 \\
\hline $\begin{array}{l}\text { COVID-19 medica- } \\
\text { tion }\end{array}$ & $\begin{array}{l}\text { Hydroxychloro- } \\
\text { quine, tocilizumab }\end{array}$ \\
\hline $\begin{array}{l}\text { Days hospitalized } \\
\text { before death }\end{array}$ & 7 \\
\hline $\begin{array}{l}\text { Proximate causes of } \\
\text { death }\end{array}$ & $\begin{array}{l}\text { Respiratory failure } \\
\text { and MSOF }\end{array}$ \\
\hline
\end{tabular}

Pathologic findings

Lung weight, left/ right $(\mathrm{g})$

Diffuse alveolar damage(DAD)

\footnotetext{
Microvascular thrombi
}

$66 \quad 57$

African American

Never smoker

\section{6}

Yes

No
None

$$
\begin{aligned}
& 630 / 910 \\
& \text { acute DAD(with } \\
& \text { scattered foci of } \\
& \text { organizing DAD) } \\
& \text { Interstitial lung } \\
& \text { disease with bron- } \\
& \text { chiectasis }
\end{aligned}
$$

Present
Patient 2

57

M

Hispanic

Never smoker

29.2

Yes

Yes

Neurologic impairment

\section{None}

None

$\begin{array}{ll}\text { NA } & \begin{array}{l}\text { Bilateral } \\ \text { Bilateral (CXR) }\end{array} \\ \text { Yes } & \text { Yes } \\ \text { VC } & \text { VC } \\ 22 & 10 \\ 1 & 0.6 \\ \text { No } & \text { No } \\ 1 & 1\end{array}$

Respiratory failure and MSOF

Patient 3

68

F

White

Current smoker

33.8

Yes

Yes

Febrile neutropenia

CAD, COPD

None

None

Respiratory failure$$
7
$$
Respiratory failure
and MSOF

$580 / 580$

$1210 / 1220$

$$
\begin{aligned}
& \text { acute DAD (with } \\
& \text { scattered foci of } \\
& \text { organizing DAD) }
\end{aligned}
$$

acute DAD and prominent reactive pneumocyte

Patient 4

76

M

White

Former pipe smoker

31.4

No

Yes

No

CAD, CHF, CKD, OSA

None

None

NA

No (CXR)

Yes

AC

16

0.8

Tocilizumab

7

$1020 / 1560$

lung injury/DAD

$$
\text { hyperplasia }
$$

Present

Present

Absent

Patients without evidence of endothelial infection or damage

Patient $5 \quad$ Patient 6

NA

No (CXR)

NA

Yes (CXR)

Yes

PC

15

0.6

Tocilizumab

0.5

No

2

5
Respiratory failure and SIRS

\section{Respiratory failure and MSOF}

$1500 / 1850$

$430 / 510$

acute DAD

$B M I$ body mass index, $S L E$ systemic lupus erythematosus, $R A$ rheumatoid arthritis, $I L D / P F$ interstitial lung disease and pulmonary fibrosis, $C K D$ chronic kidney disease, $M G U S$ monoclonal gammopathy of unknown significance, $C A D$ coronary artery disease, $C O P D$ chronic obstructive pulmonary disease, $C H F$ congestive heart failure, $O S A$ obstructive sleep apnea, $C F$ cystic fibrosis, $C V A$ cerebrovascular accident, $C T$ computed tomography, $C X R$ chest x-ray, $V C$ volume control, $P C$ pressure control, $P E E P$ positive end-expiratory pressure, FiO2 fraction inspired oxygen, $M S O F$ multi-system organ failure, $S I R S$ systemic inflammatory response syndrome 
Fig. 1 Immunofluorescence staining of ACE2, SARSCoV-2 N, CD31 in nonCOVID-19 and COVID-19 lungs. A Immunofluorescence of lung sections from human non-COVID-19 for ACE2 (magenta), SARS2-N (stain for SARS-CoV-2 nucleocapsid protein in red) and CD31 (stain for endothelium in green). DAPI serves as a nuclear DNA counterstain (blue). $\mathrm{Bar}=20 \mu \mathrm{m}$. B SARS2-N shows Type I/II pneumocyte infection. In the top row, immunofluorescence of COVID-19 decedent lung sections from patient \#3, stained for ACE2 (magenta), SARS2$\mathrm{N}$ (stain for SARS-CoV-2 nucleocapsid protein in red) and CD31 (green). DAPI serves as a nuclear DNA counterstain (blue). Positive N staining shows acute phase of diffuse alveolar damage with sloughed alveolar Type I/II pneumocytes (200× magnification). In the middle row, immunofluorescence of COVID-19 decedent lung sections from patient \#6 stained for ACE2, SARS2-N and CD31. Positive N staining shows type 1 cells with long processes along the air sacs (200× magnification). In the bottom row, zoomed insets showing the presence of viral infection in type 1 cells (630× magnification). $\mathrm{Bar}=20 \mu \mathrm{m}$
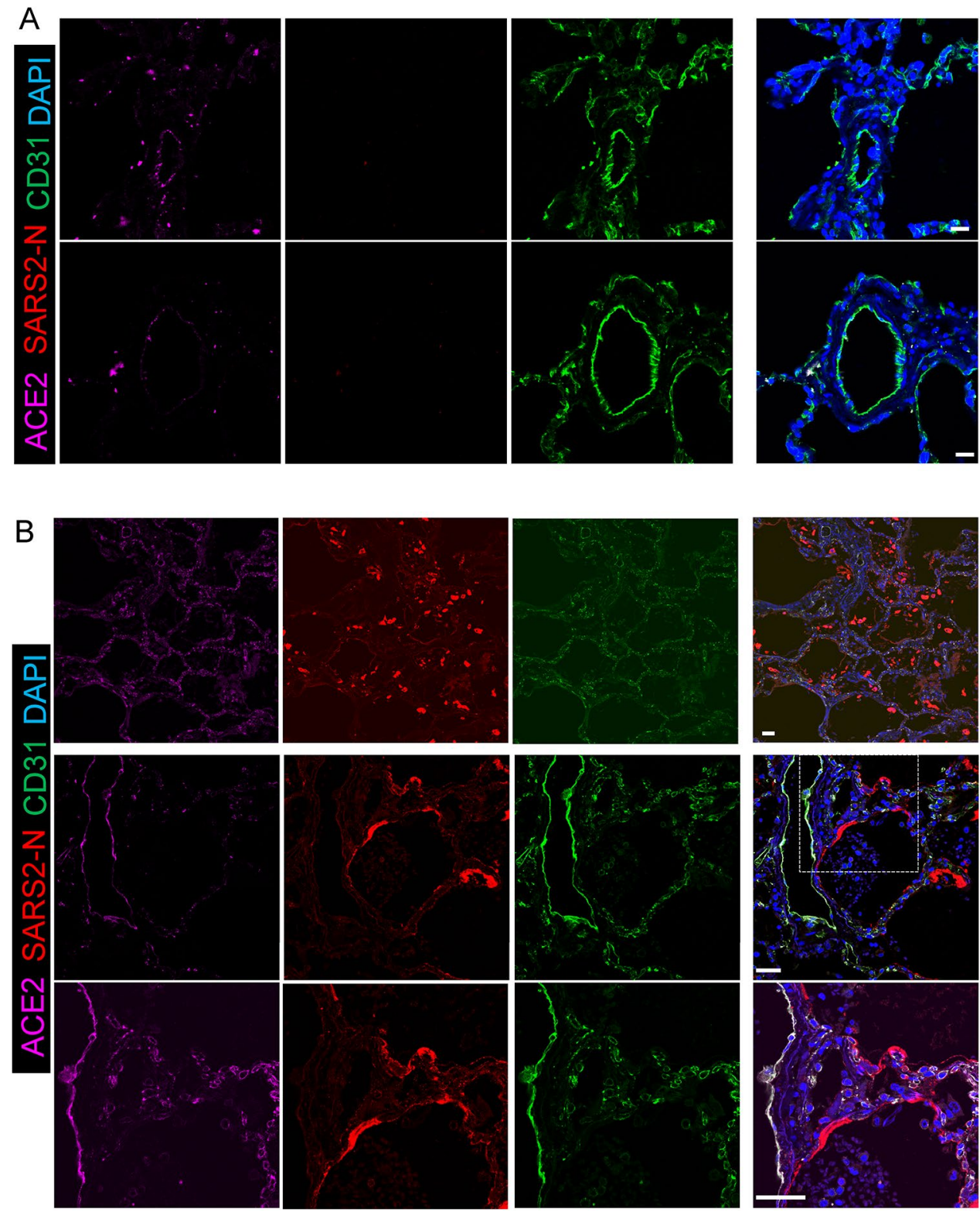

\section{No vascular infection was seen in K18-hACE2 transgenic mice}

We further evaluated the vascular infection on K18-hACE2transgenic mice, in which human ACE2 expression was driven only by the epithelial cell cytokeratin-18 (K18) promoter [8]. In control K18-hACE2 with mock infection, mouse ACE2 expression was predominantly positive in bronchial epithelial cells and type 2 pneumocytes and sparsely present on the endothelium (Fig. 3A, white arrowheads). The pattern of mouse ACE2 expression in K18-hACE2 was similar when compared to the wild-type C57/B6 strain (Suppl Fig. 6). After intranasal infection with $3.81 \times 10^{3}$ TCID50 SARS-CoV-2 at $4 \mathrm{dpi}$, SARS-CoV-2 $\mathrm{N}$ staining was dominantly distributed in alveolar pneumocytes/air sacs but not present in any sizes of vessels (Fig. 3B). Thus, human ACE2 protein expression in the endothelia is a requirement for SARS-CoV-2 infection-induced vasculopathy.

\section{ACE2 expression was low/undetectable in non-COVID-19 pulmonary/systemic endothelial cells}

The mechanism by which SARS-CoV-2 infects the endothelium was not obvious. SARS-CoV-2 cellular entry was dependent on the binding of its spike protein to ACE2 cell surface receptor, which facilitated transmembrane protease serine 2 (TMPRSS2) mediated spike protein cleavage and the induction viral-cellular membrane fusion. Though abundant on epithelial cells throughout the respiratory tract [9], 
Fig. 2 Endotheliitis is seen in tissue sections of COVID-19 lung endothelium. Immunofluorescence of COVID-19 decedent lung sections from patient \#3, stained for ACE2 (magenta), SARS2-N (stain for SARS-

CoV-2 nucleocapsid protein in red) and CD31 (endothelium in green). Zoomed insets showing the presence of viral infection in these larger vessels (diameter $>100 \mu \mathrm{m}$ ) was consistently accompanied by signs of endothelial damage and vasculopathy, including disruption of the endothelial lining, sloughing of ECs into the vascular lumen, and apoptotic ECs with distorted nuclear shape (630X magnification). Yellow arrowheads indicate positive ACE2 staining and white arrowheads indicate positive SARS2-N staining in the cytoplasm. ACE2, SARS2-N and CD31 are colocalized in an apoptotic EC. $\mathrm{Bar}=20 \mu \mathrm{m}$
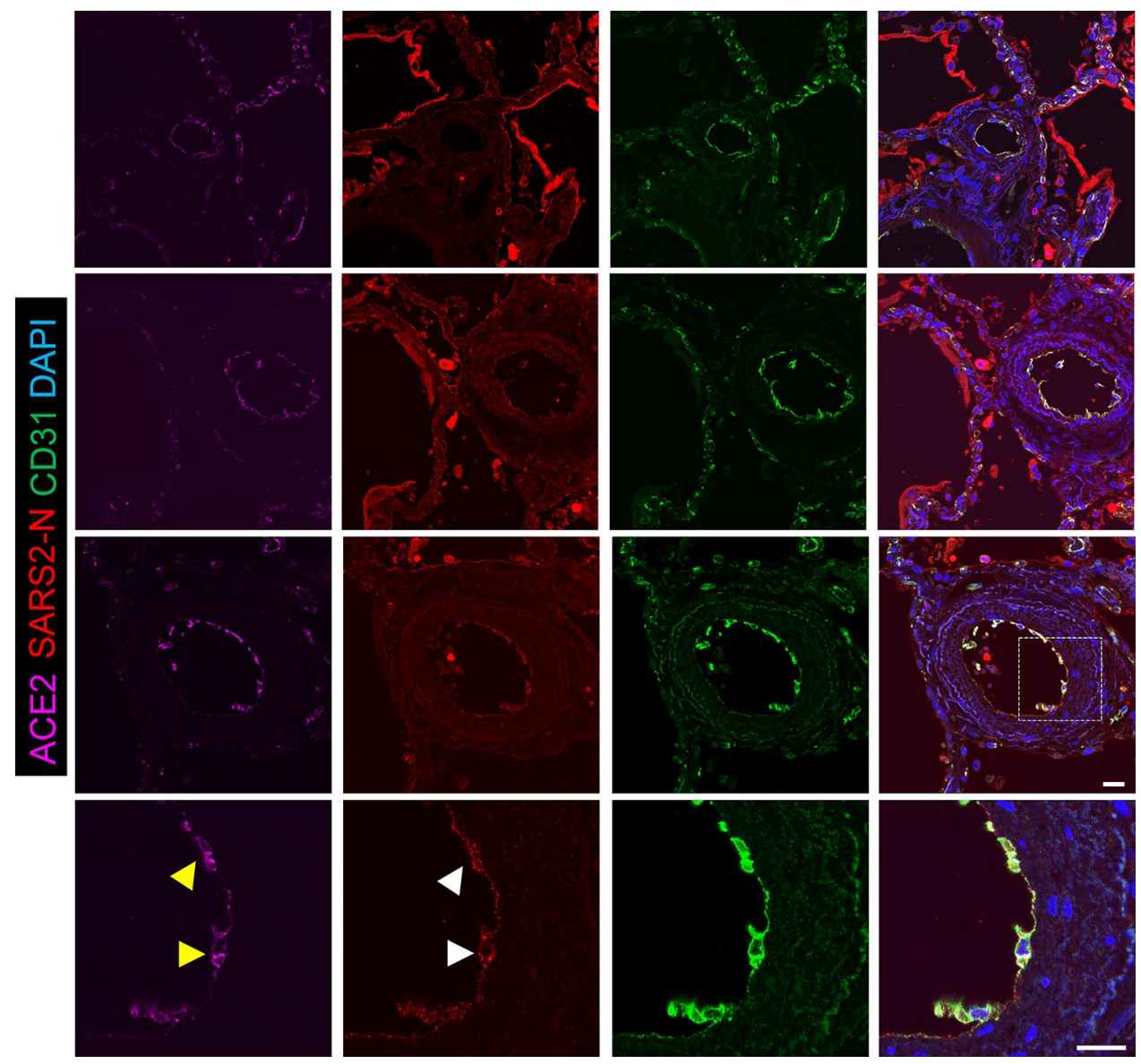

ACE2 expression was more heterogeneous and weaker on the surface of ECs of the systemic circulation $[10,11]$. Indeed, though staining with anti-ACE2 antibodies demonstrated widespread ACE2-positivity in pneumocytes in all of our post-mortem samples and in lung tissue samples from healthy controls, endothelium ACE2-positivity was more heterogeneous, and its mean fluorescence intensity was upregulated threefold in the SARS-CoV-2 infected samples (Fig. 2, Suppl Figs. 1, 2, 3, 4 5), but low/only rarely in the endothelium from non-COVID-19 samples (Fig. 1A). The observed low ACE2 expression in the endothelium of uninfected individuals was confirmed by publicly available single cell RNA-seq data, showing that ACE2 mRNA was not detectable in the endothelium of most tissues studied, particularly the pulmonary endothelium, where no ACE2 positive cells were identified among 7438 endothelial cells sampled (Suppl Tables 1, 2, 3). Similarly, screening for ACE2 mRNA expression in primary ECs sampled from multiple tissues confirmed that under unstress conditions, though ECs sampled from systemic tissues showed a broad range of $A C E 2$ expression, $A C E 2$ expression was extremely low in either pulmonary arterial or pulmonary microvascular ECs (PAECs and PMVECs, respectively) but significantly higher in the primary brain and bone ECs (Fig. 4A).

\section{Interferon alpha induced pulmonary endothelial ACE2}

It was worth noticing that viral burden positively correlated with proportions of venular endothelial cells and capillary aerocyte endothelial cells in single-cell atlases of post-mortem autopsy COVID-19 lungs [12]. Observing that ACE2 expression in the pulmonary vasculature was abundant in tissues from patients with fatal COVID-19 but none or moderately expressed in non-COVID-19 samples, we speculated that endothelial ACE2 expression was augmented in severe COVID-19 as a consequence of the host's response to infection. Severe COVID-19 was frequently complicated by an uncontrolled inflammatory response-the socalled "cytokine storm"-including the excessive release of endotheliitis-associated chemokines, such as interleukin-6 (IL6), interferon alpha and gamma (IFN $\alpha$ and IFN $\gamma$ ), C-X-C Motif Chemokine Ligand 10 (CXCL10), and Tumor Necrosis Factor alpha (TNF $\alpha$ ). High cytokine levels correlated with poor prognosis and high mortality in patients with COVID-19 [13, 14]. Given that the ACE2 gene locus included multiple interferon response element sequences and IFN $\alpha$ can induce airway epithelial $A C E 2$ expression [15], we sought to evaluate whether one or more of these cytokines 
Fig. 3 Immunofluorescence staining of mouse ACE2 and SARS2-N in tissue sections of K18-hACE2 transgenic mice. vWF (stain for endothelium in green), mouse ACE2 (red), SMA (stain for smooth muscle cell layer in green), SARS2$\mathrm{N}$ (red) and DAPI serves as a nuclear DNA counterstain (blue). A Immunofluorescence of lung sections from uninfected K18-hACE2 transgenic mice. White arrowheads indicate colocalization of vWF and mouse ACE2 staining. Bar $=20 \mu \mathrm{m}$.

B Immunofluorescence of lung sections from SARS-CoV-2-infected K18-hACE2 transgenic mice, $3.81 \times 10^{3} \mathrm{TCID}_{50}$ at 4 dpi. $B a r=20 \mu \mathrm{m}$
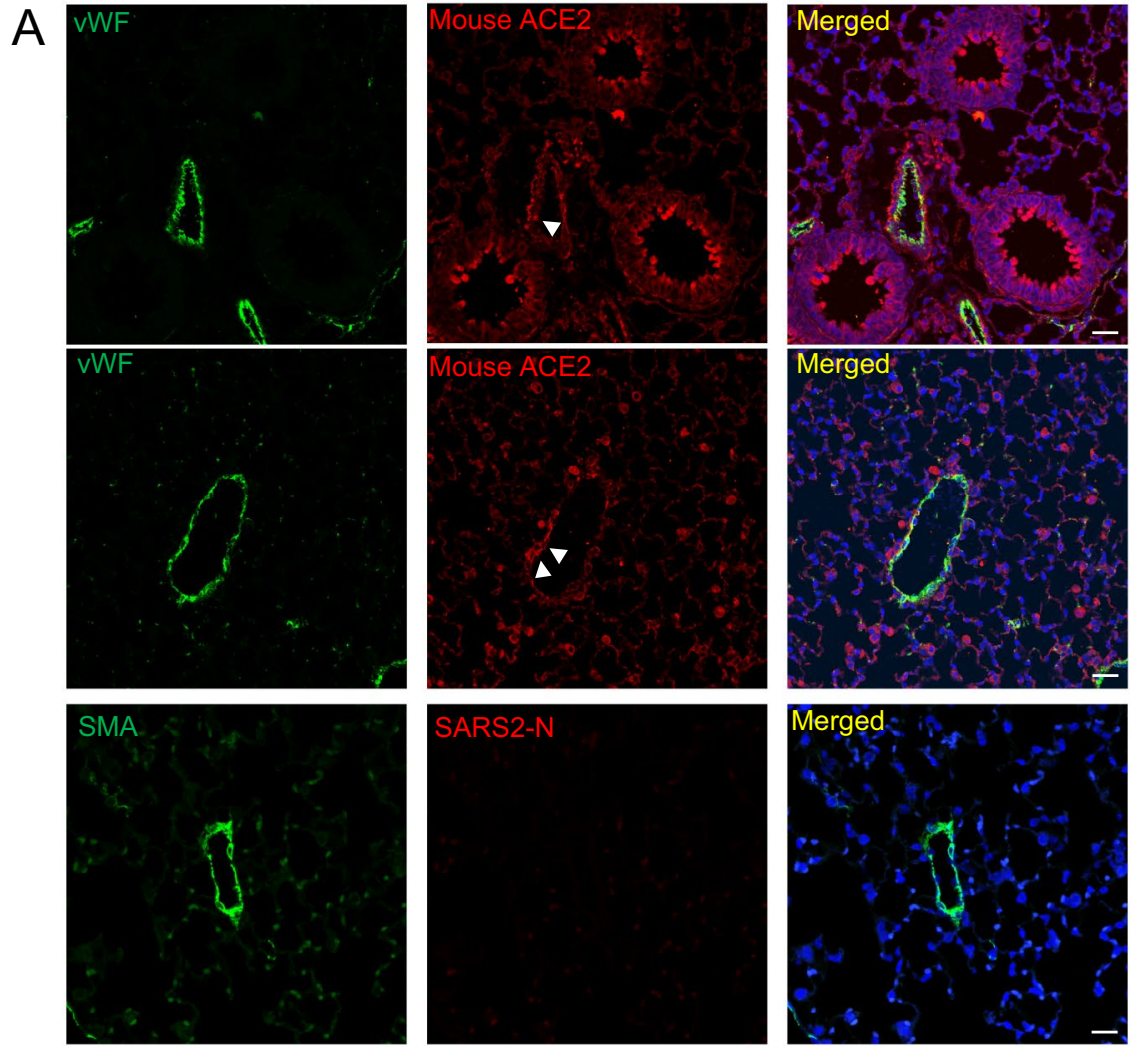

B
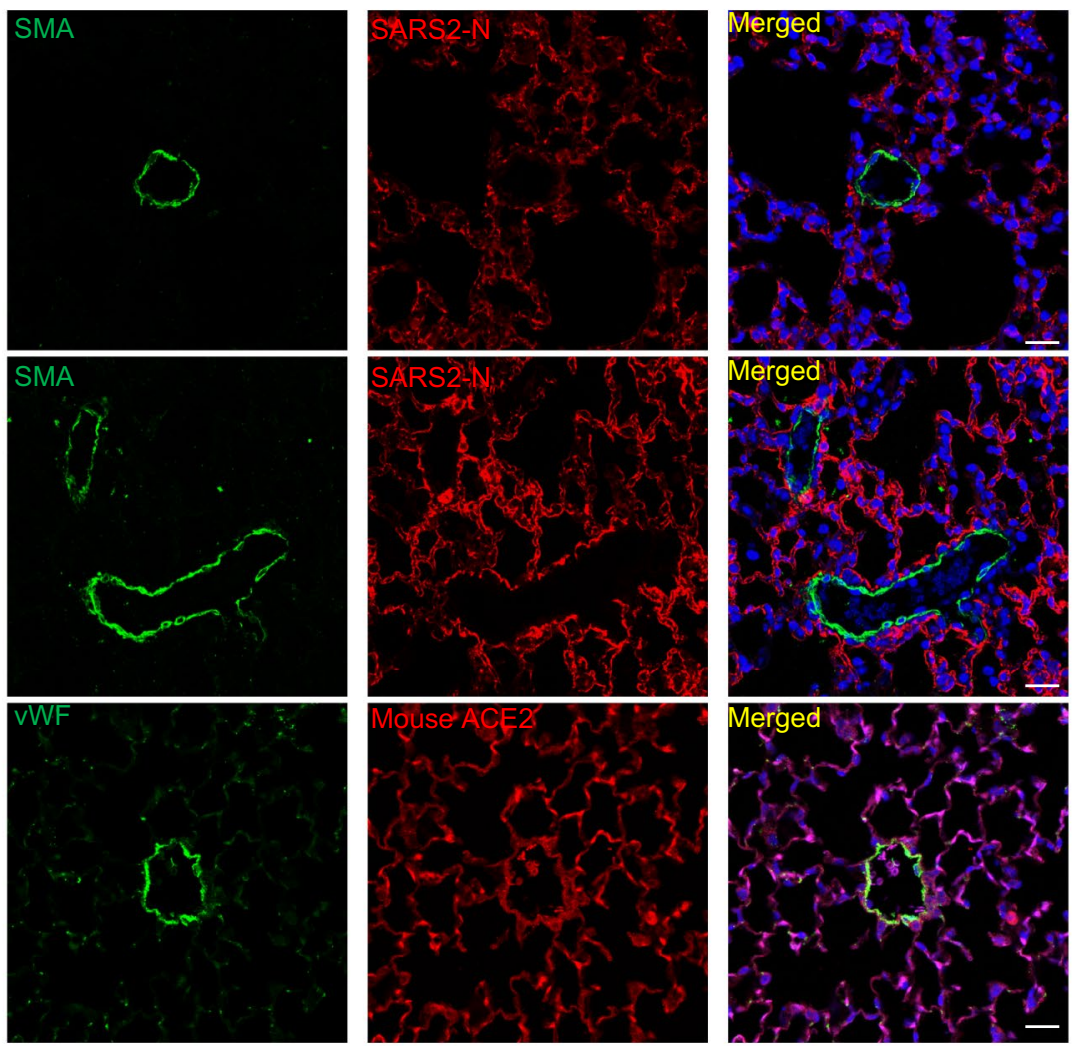
was responsible for upregulating ACE2 expression in severe COVID-19. Evaluating a range of conditions, we optimized our EC model system to ensure both PMVEC and PAEC cultures retained their fundamental physiological characteristics as a barrier and homeostasis gatekeeper, finding that $100 \%$ confluency was required for primary ECs to maintain their cubical cell shape and alignment, and form a tight junction monolayer (Suppl Fig. 7A, right panel). The addition of matrix-coating proteins, including fibronectin, rat tail or bovine collagen, and gelatin, did not enhance $A C E 2$ expression in these endothelial monolayers at the complete confluence when compared to plastic plates (Suppl Fig. 7B). Intriguingly, following the addition of a cytokine cocktail containing IL6, IFN ( $\alpha$ or $\gamma$ ), CXCL10 and TNF $\alpha$ for $6 \mathrm{~h}$, we observed sustained induction of endothelial ACE2 mRNA expression (Fig. 4B). Systematic permutation of the cytokine cocktail composition revealed that cocktails containing IFN $\alpha$ or IFN $\alpha$ alone significantly increased mRNA level of ACE2 (Fig. 4B; Suppl Figs. 8, 9). Similar patterns were observed in both PMVECs and PAECs, confirming IFN $\alpha$ as both necessary and sufficient to induce endothelial ACE2 mRNA expression. Dilution (Fig. 4C) and time-course (Fig. 4D) studies confirmed that IFN $\alpha$ was a potent, dosedependent inducer of $A C E 2$ endothelial expression, with peak expression (45-fold increase from baseline) observed at $6 \mathrm{~h}$, producing a sustained response which was observed at least $18 \mathrm{~h}$ after removal of the stimulus (by substituting for cytokine-free media at $6 \mathrm{~h}$, noted as 6-24 h, Fig. 4B). By both IF staining of PAECs (Fig. 4E) and Western blot analysis of whole-cell lysates (Fig. 5A, B), we confirmed that endothelial ACE2 induction by IFN $\alpha$ resulted in ACE2 protein synthesis, with the greatest localization to the cytoplasmic membrane (Fig. 4E, $24 \mathrm{~h}$ and 6-24 h). Consistent with prior IF staining (Fig. 4E), there was no evidence of IFN $\alpha$ influencing the expression of TMPRSS2, the spike protein cleaving enzyme (Fig. 5A). Next, we decided to measure the biological function of endothelial cells upon IFN $\alpha$ stimulation. Compared with control, IFN $\alpha$-stimulated PAECs demonstrated significantly increased leakage over 6 h. Finally, PAECs with IFN $\alpha$ resulted in shorter tube length, reduced branching points and less-organized tube networks compared with controls in Matrigel in $6 \mathrm{~h}$ (Suppl Fig. 10). Thus, IFN $\alpha$ adversely impacted on EC vessel formation, homeostasis, and barrier function.

\section{SARS-CoV-2 infected pulmonary endothelial cells}

Having demonstrated the dependence of endothelial ACE2 expression on the presence of IFN $\alpha$, we sought to determine its role in endothelial SARS-CoV-2 infection using our endothelial cell culture system and four pseudoviral constructs expressing the SARS-CoV-2 spike glycoprotein. We found that the spike protein pseudotyped viral constructs can be successfully transduced into ECs following $24 \mathrm{~h}$ stimulation with IFN $\alpha$, and with more than $50 \%$ cells expressing GFP in each experiment (Fig. 5; Suppl Fig. 11). Importantly, our results were consistent with a previous report on between-strain differences in transduction efficiency [16]: viral constructs with the d19/R682Q modifications of the spike protein known to promote the most efficient viral entry demonstrated the greatest abundance GFP-expressing ECs in our studies (Fig. 5C, D). We next confirmed the ACE2 dependent viral infection in EC cultures with infectious SARS-CoV-2. Using IFN $\alpha$-treated and untreated cells, EC cultures were infected with SARS-CoV-2 at a multiplicity of infection (MOI) of 1 or 5. By IF analysis using an antibody recognizing SARS-CoV-2 N, we found that whereas EC cells were not permissive to infection under basal conditions, ECs preconditioned with IFN $\alpha$ demonstrated a significant increase of N-positive cells by $2 \%$ (Fig. $5 \mathrm{E}, \mathrm{F}$ ), confirming that SARS-CoV-2 infection of the endothelial was dependent on induced ACE2 expression upon IFN $\alpha$ stimulation.

\section{Interferon alpha or beta induced ACE2 but not delta ACE2 in pulmonary endothelial cells}

A previous study has described a transcriptionally independent truncated ACE2 isoform-delta ACE2 (dACE2)-incapable of binding the SARS-CoV-2 spike protein and proposed that only dACE2 was the sole interferon-inducible ACE2 isoform [17-19]. Given that our initial experiments (Fig. 4A-D, Supple Figs. 7, 8 9) employed primers that do not differentiate ACE2 isoforms, we repeated our measurements using two distally-positioned primer sets that distinguish between truncated dACE2(probe 2) and full length ACE2(probe 3) (Fig. 6A; Table 2). Ct values using for full length ACE2 (probe 3) were almost consistent to those obtained using the original assay from probe 1(total ACE2). In addition, the previously published probe 2 [17] did not detect any dACE2 mRNA expression (showed an undetermined $\mathrm{Ct}$ value). This demonstrates that IFNa induces transcription of full length ACE2. We further measured ACE2 using different types of IFNs. IFN $\beta$, one of the Type I IFN family, upregulated mRNA of ACE2 up to 24-fold $(0.075 \mu \mathrm{g} /$ $\mathrm{mL})$ or 39 -fold $(0.375 \mu \mathrm{g} / \mathrm{mL})$ after $6 \mathrm{~h}$ simulation. Compared to other types of IFNs, only IFN $\alpha$ or $\beta$ induced expression of total ACE2 or full-length ACE2 (Fig. 6B) whereas the no expression of dACE was observed (data not shown). The protein expression of ACE2 was also upregulated after $24 \mathrm{~h}$ IFN $\alpha$ or $\beta$ stimulation by WB (Fig. 6C). Based on the prior condition (Fig. 5E), EC cultures were infected with SARS-CoV-2 at an MOI of 5 with or without IFN $\beta$-treatment to determine the effect of IFN $\beta$ treatment on SARS-CoV-2 susceptibility. By IF analysis using an antibody against SARS-CoV-2 N, we found that whereas EC cells were not permissive to infection under basal conditions, 


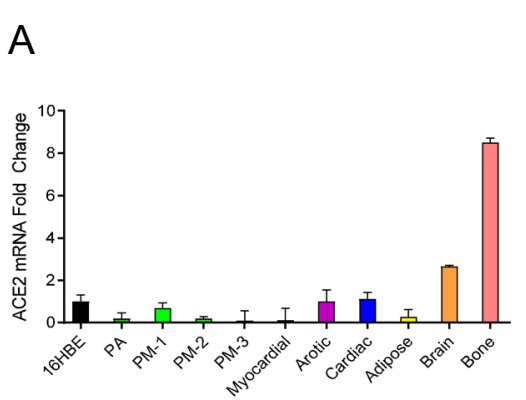

\section{B}

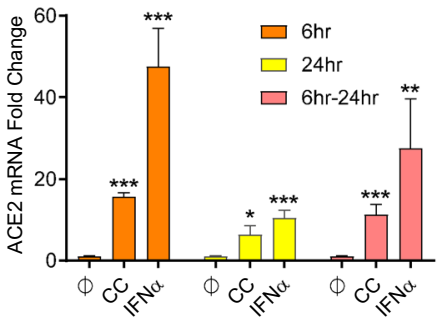

C

D
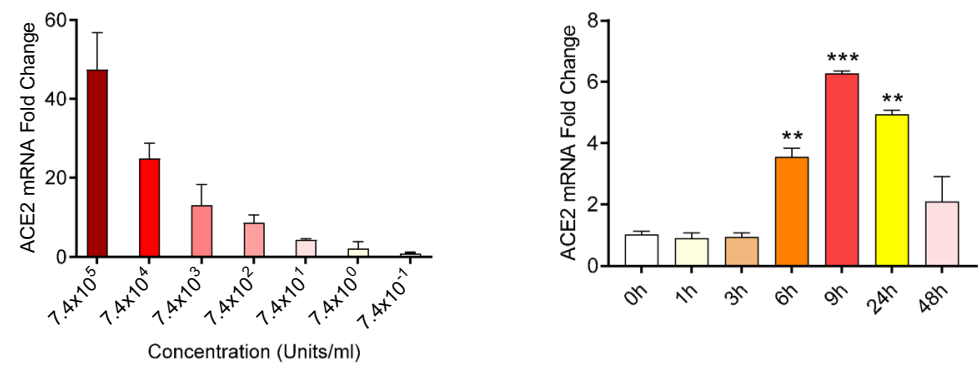

E

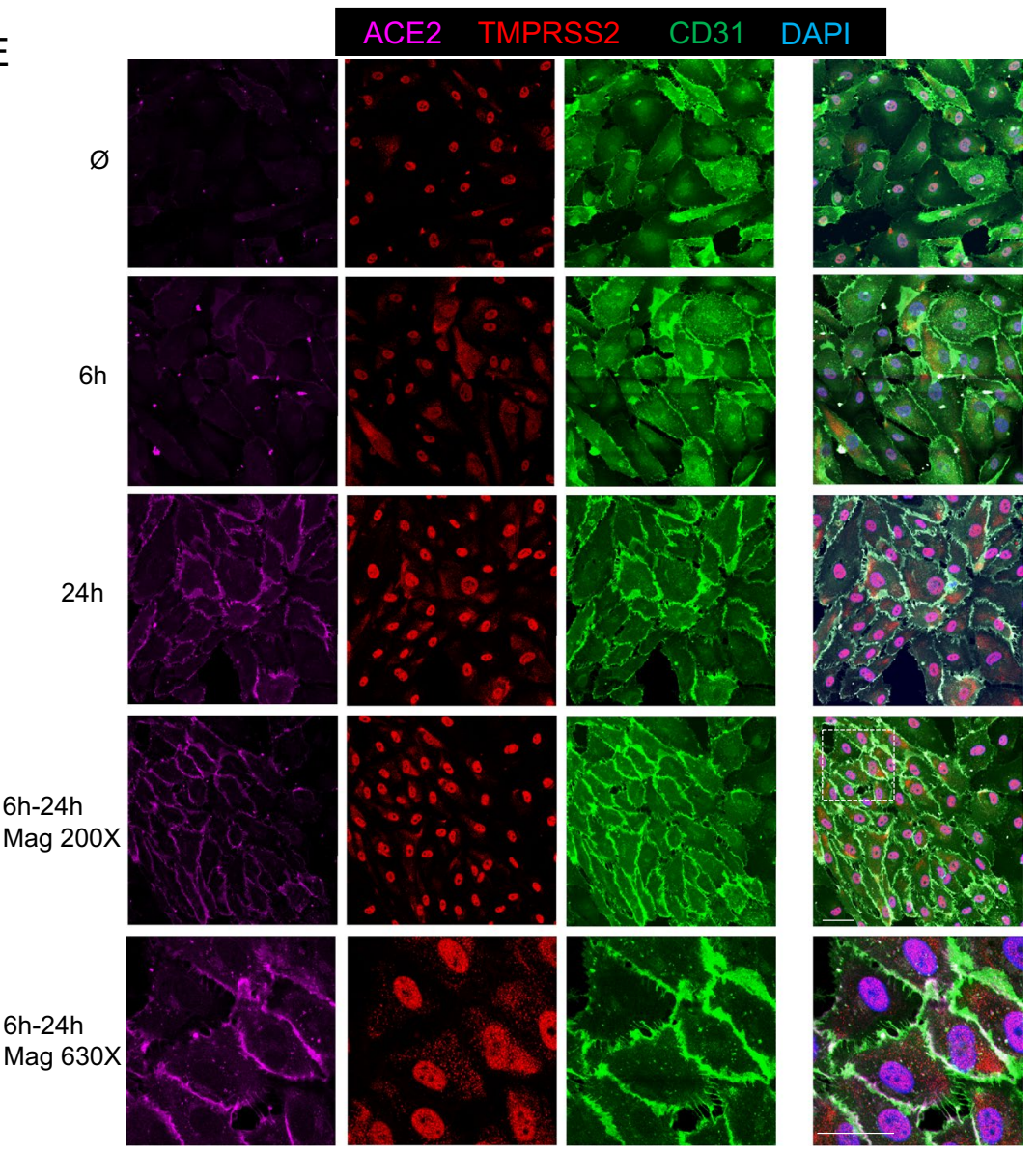


4Fig. 4 IFN $\alpha$ induces ACE2 in human primary endothelial cell cultures. A Relative mRNA expression of ACE2 in different types of human endothelial cells, including the pulmonary arterial (PA), three pulmonary microvasculature ( $\mathrm{PM}-1,-2,-3)$, myocardium (Myocardial), aorta (Aortic), cardiac microvascular (Cardiac), white adipose(Adipose), brain microvasculature (Brain) and bone (Bone). Endothelial expression is relative to that from the $16 \mathrm{HBE}$ human bronchial epithelial cell line (16HBE). Means \pm SEM are from two technical replicates. B Relative mRNA expression of ACE2 in human pulmonary arterial endothelial cells (PAECs) when incubated in cytokine-free media $(\phi)$; with a cytokine cocktail (CC-consisting of IFN $\alpha$, IFN $\gamma$, TNF $\alpha$, IL6 \& CXCL10); or with IFN $\alpha$ alone for 6, 24 and 6-24 h (a total of $6 \mathrm{~h}$ simulation and then total RNA collect in $24 \mathrm{~h}$ ). ACE2 expression level in cytokine-free media were used as control. Means \pm SEM derived from three biological replicates. $* p<0.05, * * p<0.01, * * * p<0.001$ compared to untreated control, unpaired t test. C IFN $\alpha$ dose-dependent mRNA expression of ACE2 in human PAEC. Fold-change of ACE2 expression is relative to that in untreated cells. Means \pm SEM are from two biological replicates. D Relative mRNA expression of ACE2 in human PMVEC after IFN $\alpha$ incubation for 1, 3, 6, 9, 24 and $48 \mathrm{~h}$, in a timedependent manner. Means \pm SEM are from two biological replicates. $* p<0.05, * * p<0.01, * * * p<0.001$ compared to untreated control $(0 \mathrm{~h})$, unpaired t test. E Immunofluorescence for ACE2 (magenta) TMPRSS2 (red) and CD31 (green) in PAECs treated or not with IFN $\alpha\left(7.4 \times 10^{5}\right.$ units $\left./ \mathrm{mL}\right)$ for 6,24 and $6-24 \mathrm{~h}$. Zoomed insets showing ACE2 expression on the cell membrane (630X magnification). $\mathrm{Bar}=20 \mu \mathrm{m}$

ECs preconditioned with IFN $\beta$ demonstrated a significant increase of N-positive cells (Fig. 6D). These results were further confirmed using recombinant SARS-CoV2-mNeonGreen. After $48 \mathrm{~h}$ infection, ECs preconditioned with IFN $\alpha$ or $\beta$ had increased of N-positive cells as shown by IF using an anti-N antibody (Fig. 6E). All these results implied that (at least in primary pulmonary ECs) IFN $\alpha$ and $\beta$ but not other IFNs, induced the expression of functional ACE2 capable of the binding spike protein, proviral activity of IFNs. However, in contrast to highly susceptible cells in which SARS-CoV-2 N was expressed at high amounts and was homogenously distributed at late stages of infection [20], we observed a puncate distribution pattern of $\mathrm{N}$ in the IFN-treated ECs, suggesting that viral replication in theses cells was slightly impaired. A possible explanation for this could be a balance of pro- and antiviral activity of IFN in the infected ECs.

To explore the effect of Type I IFN treatment of ECs on viruses that have been shown to be sensitive to IFN treatment and whose entry does not depend on the expression of ACE2, we infected IFN-treated ECs with Ebola virus (EBOV). EBOV is a member of the Filoviridae family and causes a severe hemorrhagic disease in humans with high fatality rates [21]. Entry of EBOV is mediated by several cell surface attachement factors and the intracellular receptor Niemann-Pick C1 (NPC1) with no dependence on ACE2 [22]. Treatment of cells with Type I IFNs renders cells resistant against EBOV infection [23] and has been shown to be beneficial for extended survival in infected nonhuman primates and Ebola virus disease patients [24, 25]. EC cultures were left untreated or treated with IFN $\alpha$ or $\beta$ for the indicated times and infected with EBOV at an MOI of 5. By IF analysis using an anti-EBOV nucleoprotein (NP) antibody, we found that ECs preconditioned with IFN $\alpha$ or $\beta$ for $24 \mathrm{~h}$ demonstrated a highly significant reduction of NP-positive cells at 24 and 48 h p.i. (Fig. 6F, G). Moreover, the EBOV infection rate in IFN $\beta$-treated cells was fourfold decreased compared to IFN $\alpha$-treated cells at $48 \mathrm{~h}$ p.i. (Fig. 6G). When cells were pretreated with IFN for $24 \mathrm{~h}$ prior to infection and and treated again at $24 \mathrm{~h}$ post infection, the reduction in EBOV infection rates was even more pronounced. As observed before, IFN $\beta$ treatment reduced the EBOV infection rate by fivefold compared to IFN $\alpha$ treatment (Suppl Fig. 12). These data show that IFNa and $\beta$ retain their antiviral potential in our cell system independent of ACE2 regulation.

\section{Discussion}

Our study suggested that endothelial damage likely occured secondary to infection in severe COVID-19. We first examined the ACE2 expression and viral infection of endothelium from six postmortem COVID-19 patient lungs using advanced microscopy. Compared with non-COVID-19 lungs, endothelial ACE2 expression was augmented in more severe COVID-19 infection as a consequence of the host's response to infection. These stainings also showed, for the first time, that the vascular distribution of SARS-CoV-2 N protein was not uniform, observed mostly in larger (diameter $>100 \mu \mathrm{m}$ ) muscularized arteries and veins but absent in arterioles and vessels smaller than $50 \mu \mathrm{m}$ in diameter. In these N-positive endothelia, endothelial damage and vasculopathy were consistently present, including disruption of the endothelial lining, sloughing of ECs into the vascular lumen, and apoptotic ECs with distorted nuclear shape (Fig. 2). Single cell RNA-seq data suggested that primary pulmonary endothelial cells were resistant to infection with SARS-CoV-2 due to the lack of ACE2 expression on the cell surface $[26,27]$. Our results strongly support these prior findings and provide the first evidence that primary endothelial cells become only susceptible to SARS-CoV-2 after IFN $\alpha$ or $\beta$ induced expression of functional ACE2 (not truncated form dACE2) (Figs. 5, 6). Our data also suggested that there was a balance between pro- and antiviral activities in the IFN-treated EC cultures. On one hand, IFNs facilitated ACE2-dependent SARS-CoV-2 entry, but on the other hand they restricted viral replication in the infected cells.

Many severe COVID-19 patients show signs of a cytokine storm, a hyperinflammatory response, which has been associated with causing the detrimental progression of COVID19. IFNs are widely considered to be anti-viral agents, 
A

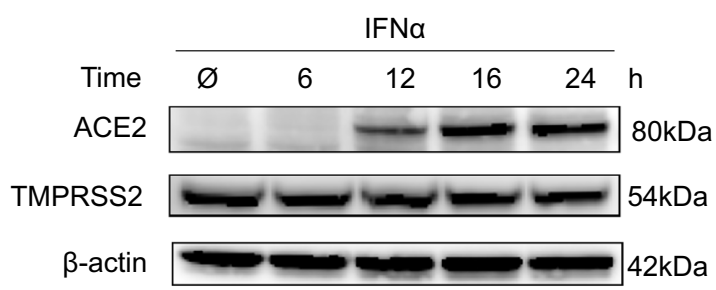

B

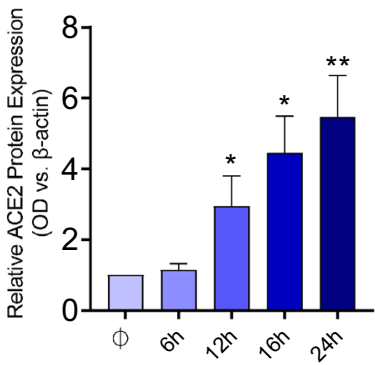

C

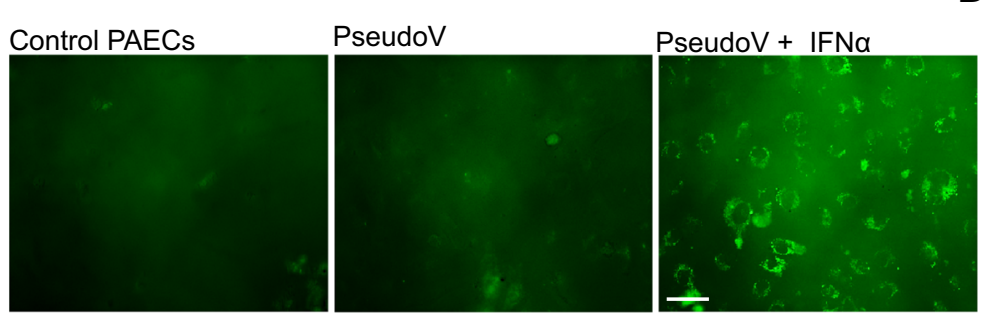

D
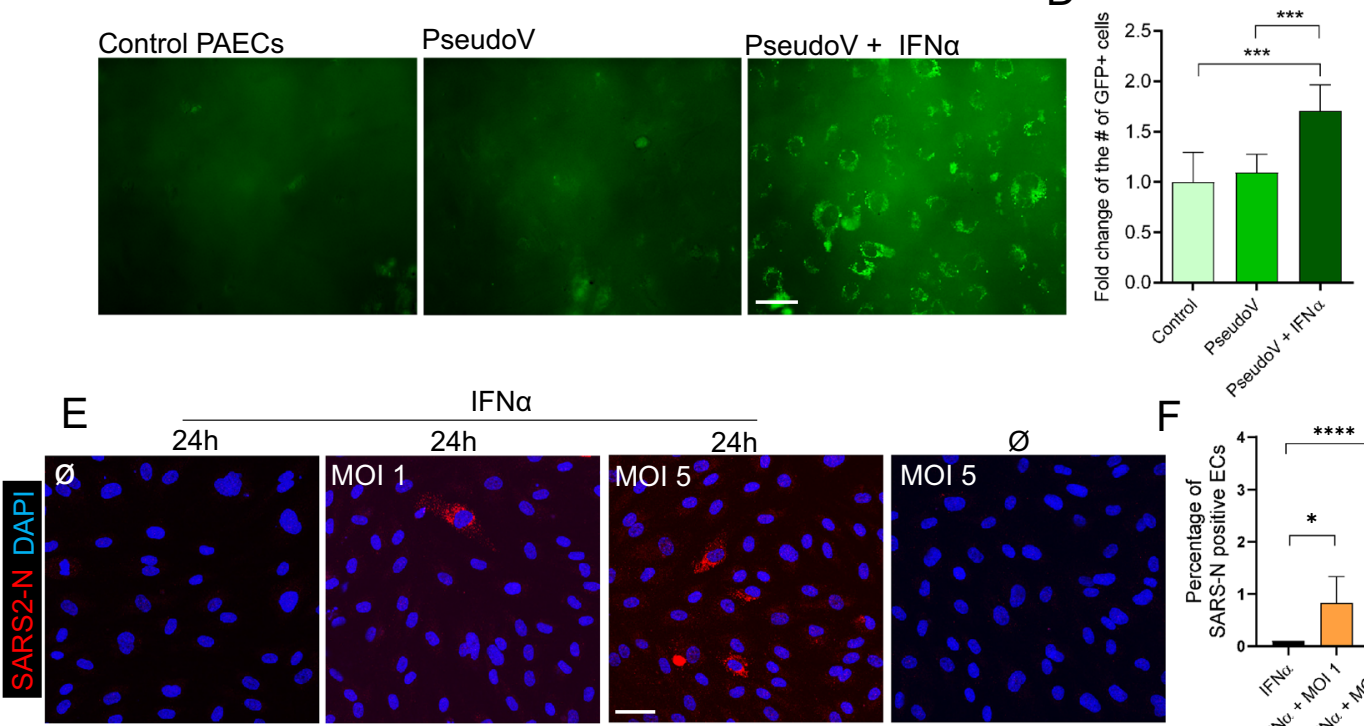

IFNa
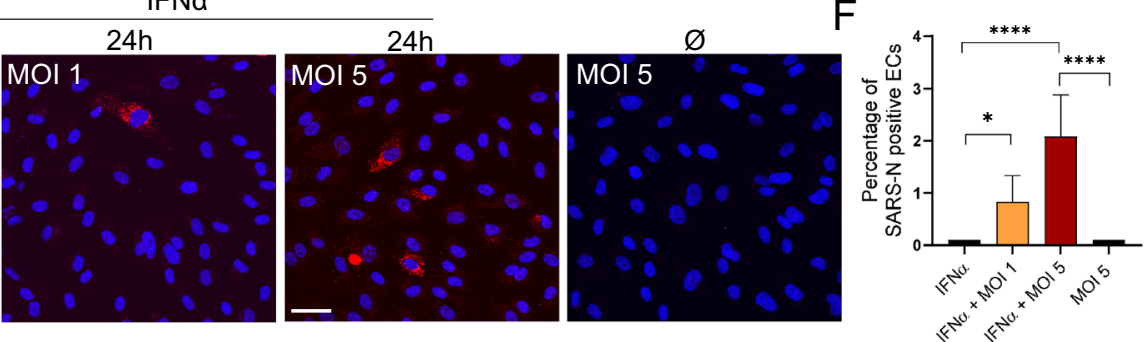

Fig. 5 SARS-CoV-2 infection in human primary arterial endothelial cells. A Representative Western blot results of ACE2 and TMPRSS2 protein expression in PAECs treated with IFN $\alpha\left(7.4 \times 10^{5}\right.$ units $\left./ \mathrm{ml}\right)$ for $6,12,16$ and $24 \mathrm{~h}$. 60ug total protein was loaded in each lane. $\beta$-actin serves as a loading control. B Optical Density (OD) quantification of ACE2 protein levels from A. Means \pm SEM are from 4 biological replicates. $* p<0.05, * * p<0.01$ compared to untreated, unpaired t test. C GFP expression in PAECs after d19/ R682Q modifications in Spike pseudoviral transduction (200× magnification). Left: PAECs only; middle: without IFN $\alpha$ stimulation but transduced with pseudovirus; right: with IFN $\alpha$ stimulation and transduced with pseudovirus. Bar $=20 \mu \mathrm{m}$ D Quantification of GFP positive cells vs. total number of cells from C. $* * * p<0.001$ compared to its control,

yet there is to date no substantial evidence of their clinical efficacy in treating COVID-19. Mechanistically, their contributions to host defense and maintenance of cellular homeostasis also remain unclear. The relationship between Type I(mainly $\alpha$ and $\beta$ ) and Type III (mainly $\lambda$ ) IFNs and COVID-19 severity remains controversial and complex. The pattern of IFN expression was measured at multiple sites, including nasopharyngeal swabs [28], bronchoalveolar lavage fluid [29], or peripheral blood [30], all of which revealed potent production of anti-viral IFN-stimulated genes (ISGs). Several studies showed that impaired IFN antiviral response in patients with severe and critical COVID-19, accompanied unpaired $t$ test. E Immunofluorescence images of viral nucleoprotein (SARS2-N, red) of PAECs infected with SARS-CoV-2 (MOI $=1$ or $5)$ at 1 day post infection (200X magnification). Left: no IFN $\alpha$ stimulation but infected at MOI 5; middle left: IFN $\alpha 24 \mathrm{~h}$ but no viral infection; middle right: IFN $\alpha 24 \mathrm{~h}$, infected at MOI 1; right: IFN $\alpha$ $24 \mathrm{~h}$, infected at MOI 5. Bar $=20 \mu \mathrm{m}$. F Quantification of the number of positive SARS2-N cells (cytoplasmic red staining along with a nuclear DAPI staining) versus the total number of cells (the number of positive nuclear DAPI staining). Means \pm SEM are calculated on six images from random fields with two biological replicates. $* p<0.001, * * * p<0.001, * * * * p<0.0001$ compared to its control, unpaired $t$ test

by high blood viral load and an excessive pro-inflammatory response [31, 32]. Nevertheless, some studies showed that dampened Type I IFNs response by autoantibodies predated SARS-CoV-2 infection and sharply increased infection prevalence in the elderly and/or severe cases [33].

In contrast to defective IFN responses, other studies have revealed caveats on the immunopathological role of IFNs and speculated on their temporal change from antiviral spreading to tissue damage at the hyperinflammatory stage [34-36], thus heightened and prolonged production of IFNs was correlated with negative clinical outcomes. For instance, classic monocytes from severe COVID-19 exhibit 
a Type I IFN-driven signature that plays a pivotal role in exaggerating inflammation in severe-COVID-19 [30]. Type III IFN induces epithelial barrier damage, causing susceptibility to lethal bacterial superinfections [37]. In a multicenter cohort study, clinical administration of Type I IFN induces favorable clinical responses during the early stage of COVID-19, while later administration is associated with increased mortality [38]. A recent in-depth analyzed study highlighted the dynamic production of IFNs in the upper or lower respiratory tract of patients during COVID-19 disease progression. SARS-CoV-2 drove the production of Type III IFN in the upper airways as an IFN protection mechanism in younger and/or patients with mild disease. Moreover, critically ill patients expressed high levels of Type I IFN in the lower airways, but reduced induction of protective ISGs [39]. Furthermore, a NIH NIAID clinical trial had found that treatment of IFN $\beta$ did not improve outcomes for hospitalized patients with COVID-19 and some patients were even associated with more adverse events and worse outcomes. These findings provided us with novel insights on the opposing roles of IFNs and reconciled some contradictory findings on IFNs. We also speculated a preexisting vascular disease or genetic predisposition may also lead to these conflicting results. In our in vitro stimulation assay, the mRNA level of ACE2 was upregulated 15 -fold by IFN $\alpha$ or 24 -fold by IFN $\beta$ stimulation as short as $6 \mathrm{~h}$. Intriguingly, after $6 \mathrm{~h}$ stimulation, IFN $\alpha$ was removed and replaced with regular culture media. The upregulated level of ACE2 mRNA was persistently upregulated for 23 -fold for $24 \mathrm{~h}$ and 7 -fold for $48 \mathrm{~h}$. These observations had clinical implications, as endothelial ACE2 could be induced by Type I IFN in an immediate and persistent manner. More extensive and temporal studies should be carried out to further evaluate the molecular mechanisms on IFN inducible ACE2 signaling axis, including existing evidence on activated downstream pathways of PD-1, NFкb [40], 40].

Our study had some limitations. First, while endothelial cell infection and damage with SARS-CoV-2 was seen in the majority of patients analyzed, only six patients were included in our analysis and larger cohorts are needed, as findings may be contributed to other factors such as preexisting endothelial damage from comorbidities such as diabetes, hypertension, a predisposed genetic susceptibility to vasculopathy. Second, IFN levels in the six patients included were not obtained. As mentioned previously, longitudinal, prospective studies are therefore needed to characterize the temporal relationship between IFN levels, viral load, and endothelial ACE2 expression in COVID-19 infected patients. Third, while our study exclusively investigated the pulmonary vasculature after infection, additional experiments in other organ systems suffering from similar complications would provide more information on the complex relationship between COVID-19 infection of the endothelium.
Finally, further in vivo and in vitro studies are needed to describe the downstream mechanism of how SARS-CoV-2 leads to endothelial cell apoptosis and denudation within the pulmonary vasculature. Recently, studies have suggested SARS-COV-2 infection may activate caspase- 3 and 8 [42, 43], which triggers cell apoptosis in lung epithelial cells, however, studies on the endothelium are needed.

Though the viral induction of interferons and their downstream response genes are an early innate host response vital to containing most respiratory viral infections, our observations suggest that the release of IFN $\alpha$ or $\beta$ in response to SARS-CoV-2 paradoxically facilitates the propagation of viral infection from respiratory epithelium to its surrounding vasculature, which in turn results in the endothelial damage that triggers the dysregulated coagulation and thrombotic complications that often drive poor patient outcomes. While the targeting of vascular IFN $\alpha$ or $\beta$ signaling as a potential therapeutic strategy to prevent disease progression in patients with severe COVID-19 will require additional study, our findings raise a more pressing concern and reveal the mechanism regarding current treatment guidelines that propose the use of exogenous recombinant IFN $\alpha$ or $\beta$ for the treatment of severe infection. Such recommendations should urgently be revisited.

\section{Materials and methods}

\section{Study approval}

The autopsy portion of this study was approved by the Institutional Review Board of Brigham and Women's Hospital. The pseudoviral use of this study was approved by the Institutional Biosafety Committee of Boston Children's Hospital.

\section{Clinical subjects}

All autopsies were performed by one of the authors (RFP) at Brigham and Women's Hospital on patients diagnosed with SARS-CoV-2 infection by nasopharyngeal swab RT-PCR either upon clinical presentation or post-mortem. The lungs were weighed, inflated with $10 \%$ neutral buffered formalin, sectioned and embedded in paraffin via standard procedures. Epidemiological, clinical, laboratory and radiological data were obtained from the electronic medical records.

\section{Immunohistochemistry}

Postmortem lung tissues obtained from patients with COVID-19 were fixed in 10\% neutral buffered formalin, paraffin embedded, sectioned at $5 \mu \mathrm{m}$, dewaxed, and rehydrated by standard procedures. Paraffin slides were placed into the vacuum at 60 degree Celsius for $20 \mathrm{~min}$ 

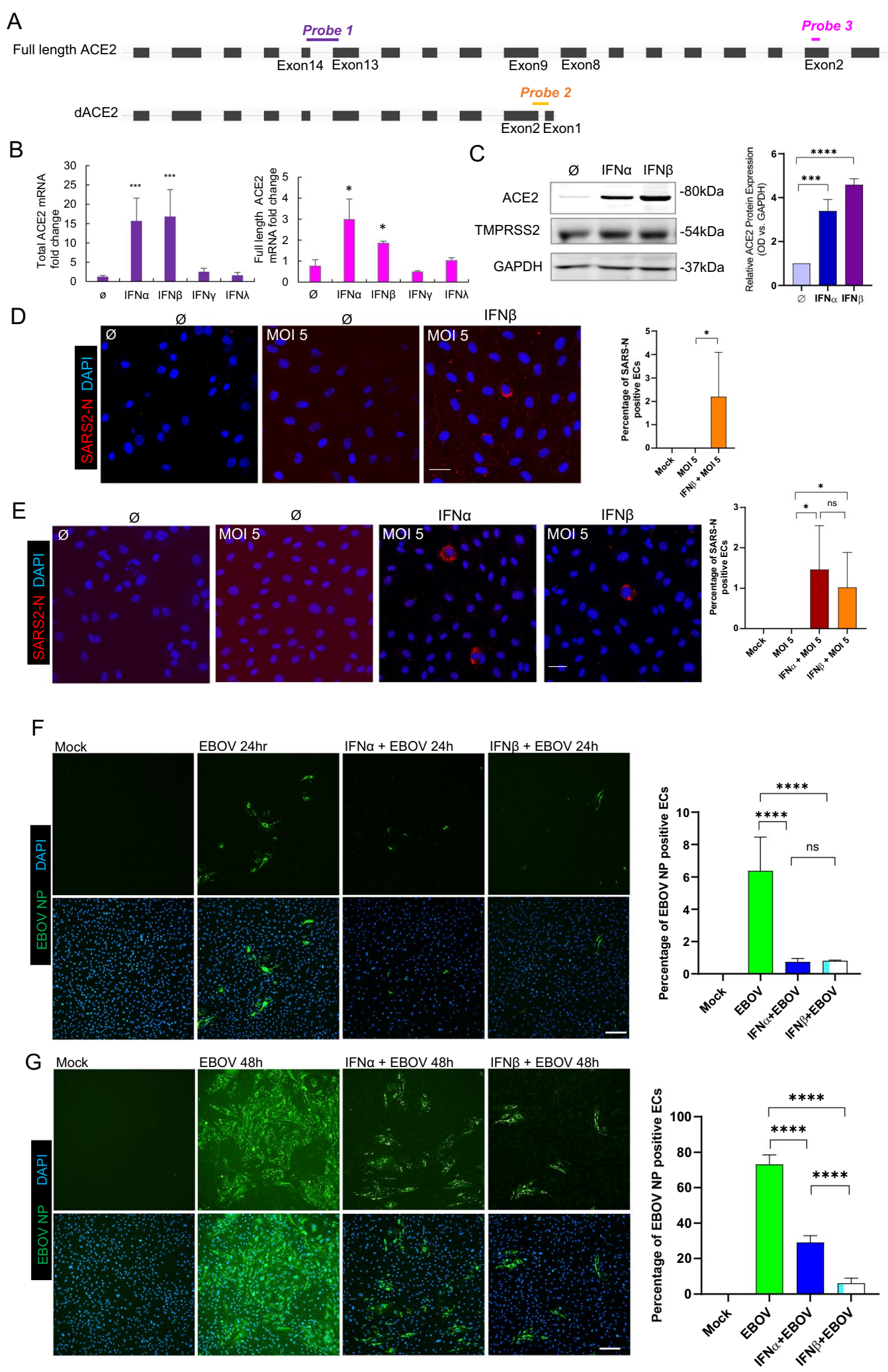

to achieve dewaxing. After, they were rehydrated by placing samples into Xylene and ethanol washes. Samples were then immediately placed into boiling $1 \times$ Citrate
Buffer (pH of 6.0) (C2488, Milipore/Sigma-Aldrich) for $10 \mathrm{~min}$ and following this allowed to sit in room air for $1 \mathrm{~h}$. When at room temperature, samples were washed with $1 \mathrm{X}$ 
4Fig. 6 SARS-CoV-2 or Ebola virus infection of human primary PAECs after IFN $\alpha$ or $\beta$ stimulation. A Schematic representation of ACE2 and delta ACE2(dACE2) transcripts and the position of the three PCR probes to generate wildtype ACE2, delta(d)ACE2 and full length ACE2 amplicons. B Expression of total ACE2 (left) and expression of full length ACE2 (right) in three PMECs after treated with IFN $\alpha, \beta, \gamma, \lambda$ for $6 \mathrm{~h}$. C Representative Western blot results of ACE2 and TMPRSS2 protein expression in PAECs treated with IFN $\alpha$ or $\beta$ for $24 \mathrm{~h} .60 \mu \mathrm{g}$ total protein was loaded in each lane. GAPDH serves as a loading control. Optical Density (OD) quantification of ACE2 protein levels vs GAPDH. Means \pm SEM are from three biological replicates. D Immunofluorescence images of viral nucleoprotein (SARS2-N, red) of PAECs infected with SARS-CoV-2 (MOI=5) at 1 day post infection (200X magnification). Left: Mock; middle: no IFN $\beta$; right: IFN $\beta 24 \mathrm{~h}$. Bar $=20 \mu \mathrm{m}$. E Immunofluorescence images of viral nucleoprotein (SARS2-N, red) of PAECs infected with recombinant SARS-CoV-2-mNeonGreen $(\mathrm{MOI}=5)$ at 1 day post infection (200× magnification). Left: Mock; middle left: no IFN; middle right: IFN $\alpha$; right: IFN $\beta 24$ h. Bar $=20 \mu \mathrm{m}$. F Immunofluorescence images of viral nucleoprotein (EBOV NP, green) of PAECs at $24 \mathrm{~h}$ post infection or at $48 \mathrm{~h}$ post infection $(\mathbf{G})$. Cells were pretreated with IFNs for $24 \mathrm{~h}$ and infected with wildtype Ebola virus (EBOV) $(\mathrm{MOI}=5)$ Left: Mock; middle left: no IFN; middle right: IFN $\alpha$; right: IFN $\beta$. Bar $=50 \mu \mathrm{m}$. Quantification (both $\mathbf{D}, \mathbf{E}, \mathbf{F}$ and $\mathbf{G}$ ) of the number of positive SARS2-N or EBOV NP (cytoplasmic positive staining along with a nuclear DAPI staining) versus the total number of cells (the number of positive nuclear DAPI staining). Means \pm SEM are calculated on six images from random fields with two technical replicates. $* p<0.05, * * * p<0.001$ and $* * * * p<0.0001$ compared to its control, unpaired t test

PBST(Tween20) and then blocked with donkey blocking serum for $1 \mathrm{~h}$. Primary antibodies of interest, including sheep anti-CD31 (1:100; AF806, R\&D systems), rabbit anti- SARS-N protein (1:500; 200-401-A50 Rockland), goat anti-ACE2 (1:50; AF933, R\&D Systems), rabbit anti-vWF(1:300; Agilent A0082) were then applied at 4 degrees Celsius and allowed to incubate overnight. The next day, samples were washed five times for $10 \mathrm{~min}$ each in PBST and donkey blocking serum was applied at room temperature for $1 \mathrm{~h}$. Secondary conjugate antibodies, donkey anti-sheep Alexa Fluor 488 (1:250; 713545-003, Jackson Immunoresearch), donkey anti-rabbit Alex Fluor 555 (1:250; 711-165-152, Jackson Immunoresearch) and donkey anti-goat Alexa fluor 647 (1:250; 705605-147, Jackson Immunoresearch) were applied for $6 \mathrm{~h}$ at room temperature. Slides were then washed five times with PBST again for 10 min each and mounted with DAPI (AF806, Vector Laboratories) to prepare for imaging. Following immunohistochemistry, the sections were imaged using Zeiss Confocal Z880 Airyscan microscopy.

K18-hACE2 transgenic mouse slides were provided by Dr. Hongpeng Jia (John Hopkins University). Paraffin slides were treated the same way as human slides. Primary antibodies of interest, including rabbit anti-vWF (1:200; A008202-5, Agilent), rabbit anti- SARS-N protein (1:500; 200-401-A50 Rockland), rat anti-mouse ACE2 (1:50;
MAB3437, R\&D Systems) and SMA-Alexa 647(1:50, sc-32251, Santa Cruz).

\section{Cell culture}

Isolation of human ECs from adipose, bone, and myocardium tissues: Endothelial cells were isolated from normal human subcutaneous white adipose tissue, iliac crest corticocancellous bone tissue, and ventricular myocardial tissue. All these human samples were deidentified and discarded tissues obtained during clinical-indicated procedures in accordance with Boston Children's Hospital Institutional Review Board-approved protocols. Tissues were minced and enzymatically (collagenase and dispase) digested for $2 \mathrm{~h}$ at $37^{\circ} \mathrm{C}$. Erythrocytes were lysed with RBC lysis buffer (New England Biolabs, Cat No. 420301). White adipose-, bone-, and myocardial-ECs were isolated by magnetic activated cell sorting (MACS) using CD31-coated magnetic beads (Dynabeads, Invitrogen, Cat No. 11155D). Isolated ECs were cultured on $1 \%$ gelatin-coated plates using EC medium: EGM-2 (except for hydrocortisone; PromoCell, Cat No. C22111) supplemented with $10 \%$ GenClone FBS (Genesee, Cat No. 25-514) and $1 \times$ glutamine-penicillin-streptomycin (GPS, ThermoFisher, Cat No. 10378106). Three human pulmonary microvascular endothelial cells are purchased from PromoCell(C-12282). Human pulmonary artery endothelial cells (3100), human Aortic Endothelial cells (6100), human cardiac microvascular endothelial cells (6000), human brain microvascular endothelial cells (1000) are purchased from ScienCell and maintained in Endothelial Cell Medium (ECM, 1001, ScienCell), supplemented with 5\% fetal bovine serum (0025, ScienCell), 1\% penicillin/streptomycin (P/S, 0503, ScienCell), and Endothelial Cell Growth Supplement (ECGS, 1052, ScienCell) and incubated at $37^{\circ} \mathrm{C}$ in an incubator with an atmosphere of 5\% CO2/95\% air. Cells were digested with $0.25 \%$ trypsin EDTA (MT25053CI, Corning) and passaged when reaching $90 \%$ confluency. All experiments were performed with ECs before passage six. Gelatin (1:2, Sigma G1393), Type 1 bovine collagen (1:60, Advanced BioMatrix, Cat \#5005), Cultrex Bovine Collagen I (1:100, R\&D 3442-050-01), fibronectin (1:100, Sigma F1141) are diluted in 1X PBS and used for coating at least $1 \mathrm{~h}$ at room temperature.

\section{Cytokine stimulation}

The day before, coat the culture plates with $1 \%$ gelatin for $1 \mathrm{~h}$ and seed a substantial number of cells on the coated culture plates. The next day, EC must form a monolayer to proceed with the stimulation. The concentration of cytokine cocktail are made of recombinant human IFN-alpha A (alpha 2a) $\left(7.4 \times 10^{5}\right.$ units/mL, R\&D cat\# 11100-1) IFN $\gamma$ (0.5ug/mL, Peprotech \#300-02), TNFa(0.1 $\mu \mathrm{g} / \mathrm{mL}, \mathrm{R} \& \mathrm{D}$ 
Table 2 Sequences and amplicons info of qPCR probe sequences for total ACE2, dACE2 and full length ACE2

\begin{tabular}{|c|c|c|c|c|c|c|}
\hline Name & Forward & Reverse & Probe sequence & $\begin{array}{l}\text { Ampli- } \\
\text { con size } \\
\text { (bp) }\end{array}$ & Comment & References \\
\hline Probe 1 & $\begin{array}{l}\text { GCCACTGCTCAACTA } \\
\text { CTTTG }\end{array}$ & $\begin{array}{l}\text { GCTTATCCTCACTTT } \\
\text { GATGCTTTG }\end{array}$ & $\begin{array}{l}\text { ACTCCAGTCGGTACT } \\
\text { CCATCCCA }\end{array}$ & 124 & Total ACE2 & Commercial (IDT) \\
\hline Probe 2 & $\begin{array}{l}\text { GGAAGCAGGCTGGGA } \\
\text { CAAA }\end{array}$ & $\begin{array}{l}\text { AGCTGTCAGGAAGTC } \\
\text { GTCCATT }\end{array}$ & $\begin{array}{l}\text { AGGGAGGATCCTTAT } \\
\text { GTG }\end{array}$ & 73 & Only dACE2 & Onabajo et al \\
\hline Probe 3 & $\begin{array}{l}\text { ATAATGCTGGGGACA } \\
\text { AATGG }\end{array}$ & $\begin{array}{l}\text { TGTTCAACCGTTTGC } \\
\text { TCTTG }\end{array}$ & $\begin{array}{l}\text { TCCACACTTGCCCAA } \\
\text { ATGTA }\end{array}$ & 346 & Only full length & Self-design \\
\hline
\end{tabular}

210-TA-020), IL6(0.1 $\mu \mathrm{g} / \mathrm{mL}$, Peprotech \#200-06), CXCL10 $(0.1 \mu \mathrm{g} / \mathrm{mL}$, Peprotech \#300-12). After incubation cytokines with a determined time point, total RNA will be collected and proceed with cDNA reverse transcription.

\section{Pseudoviral tranduction}

The SARS-CoV-2 S protein cDNA was used to pseudotyped human immunodeficiency virus (HIV) expressing an enhanced green fluorescence by using previously described methods [16]. A vesicular stomatitis virus G (VSV G) protein pseudotyped HIV expressing eGFP was used as positive control for viral transduction. The day before viral transduction, the culture plates (ibidi 3 well insert Cat\# 80366) were coated with $1 \%$ gelatin for $1 \mathrm{~h}$ and seed 12,000 cells on the coated culture plates. The next day, EC must form a monolayer to proceed with the IFN $\alpha$ stimulation. IFN $\alpha(1: 120)$ diluted in full EC media and incubated on cells for $24 \mathrm{~h}$. Wash cells with 1 XPBS three times and dilute any of three pseudovirus 1:5 ratio in serum free and ECGS free medium. Incubate pseudovirus solution on cells for $2 \mathrm{~h}$. Remove the viral solution, wash $3 \mathrm{X}$ with $1 \mathrm{X}$ PBS, and change back to full EC medium. GFP signal is acquired after $24 \mathrm{~h}$ using Zeiss Axio Observer Z1 Inverted Epifluorescence Microscope. Live cellular DAPI staining (Invitrogen R37605) is added to the medium to counterstain the nuclei.

\section{Virus propagation and titration}

SARS-CoV-2 (isolate USA_WA1/2020) was kindly provided by CDC's Principal Investigator Natalie Thornburg and the World Reference Center for Emerging Viruses and Arboviruses (WRCEVA). Recombinant SARS-CoV-2 expressing mNeonGreen (SARS-CoV-2-mNG) was kindly provided by Pey-Yong Shi, University of Texas Medical Branch, Galveston and the World Reference Center for Emerging Viruses and Arboviruses [44]. This virus is based on SARS-CoV-2 isolate SARS-CoV-2 isolate USA_WA1/2020. EBOV (isolate Mayinga) was kindly provided by Heinz Feldmann, NIH NIAID Rocky Mountain laboratories. All virus stocks were propagated in Vero E6 cells (ATCC CRL-1586) cultured in Dulbecco's modified Eagle's medium (DMEM) supplemented with $2 \%$ fetal calf serum (FCS), penicillin (50 U/ $\mathrm{ml})$, and streptomycin $(50 \mathrm{mg} / \mathrm{mL})$. Viral stocks were purified by ultracentrifugation through a $20 \%$ sucrose cushion at $80,000 \times g$ for $2 \mathrm{~h}$ at $4{ }^{\circ} \mathrm{C}$ as described before [20]. Viral titers were determined in Vero E6 cells by tissue culture infectious dose 50 (TCID50) assay using the Spearman and Kärber algorithm. All work with SARS-CoV-2 and EBOV was performed in the biosafety level 4 (BSL4) facility of the National Emerging Infectious Diseases Laboratories at Boston University, Boston, MA following approved SOPs.

\section{Viral infection of slides}

PAECs were seeded at a density of $5.2 \times 10^{4}$ cells per well in 8-well ibidi slides (Cat\# 80,826) in full ECM (ECM, 1001, ScienCell). For infection, cell supernatants were removed and replaced with $120 \mu \mathrm{L}$ of ECM without FBS or growth supplement containing SARS-CoV-2, SARS-CoV-2-mNG or EBOV. Cells were infected at an MOI of 1 or 5 for $2 \mathrm{~h}$. Infection medium without virus was used as mock control. After a $2 \mathrm{~h}$ incubation period at $37^{\circ} \mathrm{C}$ in $5 \% \mathrm{CO}_{2}$, inoculum was removed, and cells were washed once with PBS. $300 \mu \mathrm{l}$ of full ECM were added per well, and cells were incubated at $37{ }^{\circ} \mathrm{C}, 5 \% \mathrm{CO}_{2}$ until fixation. For additional treatment with IFNs, IFNa or $\beta$ was added into corresponding wells at $24 \mathrm{~h}$ post infection. For fixation, cell supernatants were removed, and cells were washed once with PBS and fixed in $10 \%$ formalin for at least $6 \mathrm{~h}$ and removed from the BSL-4 laboratory in accordance with approved SOPs. Briefly, EBOV staining was performed using anti-EBOV-NP (IBT Bioservices, 1:200 overnight) and followed with goat-antirabbit-AF488 (Invitrogen 1:200, $1 \mathrm{~h}$ ) and the mounting media containing DAPI.

\section{RNA extraction and qPCR}

RNA of multiple primary human endothelial cells was extracted using RNeasy Mini Kit (74,106, Qiagen), and reverse transcribed with High-Capacity cDNA Reverse Transcription Kit (4,374,966, Applied Biosystems). 
Quantitative RT-PCR was performed on QuantSudioTM 7 Flex Real-time System (Applied Biosystems) with TaqMan probes predesigned by Integrated DNA technologies. Relative expression level of ACE2 was calculated based on the standard $2-\Delta \Delta$ CT method using GAPDH as a reference gene. Gene expression comparisons were performed using unpaired t-test.

\section{Western blot}

Total protein $(60 \mu \mathrm{g})$ extracted from human pulmonary artery endothelial cells was separated by $4-12 \%$ SDS-PAGE (NP0321, Invitrogen), transferred to a PVDF membrane, and immunoblotted with goat polyclonal ACE2 antibody (AF933, R\&D), rabbit anti-TMPRSS2 antibody (ab92323, Abcam) or mouse anti-beta Actin antibody (ab49900, Abcam) as a loading control. Anti-goat, anti-rabbit and antimouse HRP antibodies were purchased from Abcam. Protein bands were detected using the LAS-4000 luminescent imaging system (Fujifilm Life Science) and quantified with Image $\mathrm{J}$ software. Protein expression comparisons were performed using unpaired t-test.

\section{Fluorescein isothiocyanate (FITC)—dextran permeability assay}

$0.4 \mu \mathrm{m}$-pore inserts in a 24-well plate (Costar \#38024) were purchased. Before the experiment, inserts were coated with $1 \%$ gelatin in room temperature for 1 h. $2.5 \times 10^{4}$ PAECs were plated onto the collagen-coated inserts in a $37{ }^{\circ} \mathrm{C} / 5 \%$ $\mathrm{CO} 2$ tissue culture incubator. After overnight incubation, IFN $\alpha(500 \mathrm{U} / \mu \mathrm{L})$ was added on apical side. After 24 -h IFN $\alpha$ treatment, a solution of $1 \mu \mathrm{g} / \mathrm{mL}$ FITC-Dextran (MW: $70 \mathrm{kDa}$; Sigma) in $100 \mu \mathrm{L}$ full ECM was added in the inserts and $600 \mu \mathrm{L}$ full ECM in the lower chamber. Fluorescence intensity was detected every hour up to $6 \mathrm{~h}$ by using the Microplate Reader (Tecan Spark multimode microplate reader; Switzerland) with excitation and emission wavelengths of 492 and $520 \mathrm{~nm}$, respectively.

\section{Matrigel angiogenesis assay}

Matrigel (356,231, Corning) was thawed on ice overnight, loaded $10 \mu \mathrm{L}$ each well on $\mu$-Slide Angiogenesis glass bottom plate (Ibidi product \#81507, Munich, Germany), and incubated for $1 \mathrm{~h}$ at $37^{\circ} \mathrm{C}$ for polymerization. $3 \times 10^{3}$ PAEC alone or $3 \times 10^{3}$ PAEC mixed IFN $\alpha$ in $50 \mu \mathrm{L} \mathrm{ECM}$ were dispersed on each Matrigel-coated well. Images were captured every hour up to $4 \mathrm{~h}$ by using a Leica Inverted microscope. Total tube lengths, total branching points and number of loops were quantified using AngioTool and ImageJ.

\section{Statistics}

Statistical analyses were performed using GraphPad Prism 7. Unpaired Student's t-test was used for two-group comparisons. All stimulated groups are compared to the relevant control group. More details are described in the figure legends.

Supplementary Information The online version contains supplementary material available at https://doi.org/10.1007/s10456-021-09823-4.

Acknowledgements We thank Dr. Marc Johnson for the kind gift of pseudoviral SARS-CoV-2 particles. We thank Dr. Ruobing Wang for critical review/discussion of this work. We thank Drs. Jin-Ah Park, Jeffrey J. Fredberg, Ramaswamy Krishnan and Darrell Kotton for technical advice and discussions. We thank Michelle Siciliano, Jacob Plaisted and John Grzyb for their dedication, expertise and professionalism in assisting in the performance of the autopsies of the COVID-19 patients. We thank all the patients and their families involved in this study as well as the numerous doctors, nurses, and civilians working together to fight against COVID-19.

Author contributions TK, YH, HK, JK, JO, AJH and KY planned and performed experiments. TK, YH, HK and KY were responsible for data analysis. TK, YH, EM, HJ, RFP, BAR and KY provided intellectual input. XH, JMM, YF and RFP provided reagents. BAR and KY conceived the study. TK, HY, JO, EM, BAR and KY wrote the manuscript. The order of the co-first authors was decided depending on the combined contribution of intellectual input and experiments performed.

Funding This work was supported by NIH NHLBI 1R01HL15010601, Parker B Francis Fellowship and ATS/PHA Aldrighetti Research Award for Young Investigators (to KY); NHLBI 5T32HL00763336 (to TK); Fast Grants, the Evergrande COVID-19 Response Fund Award from the Massachusetts Consortium on Pathogen Readiness, NIH NCATS Grant UL1TR001430, R21 AI135912 (to EM); NIH 3R21AI149321-01S1, R21AI149321, R01AI148446 (to HJ).

Open Access This article is licensed under a Creative Commons Attribution 4.0 International License, which permits use, sharing, adaptation, distribution and reproduction in any medium or format, as long as you give appropriate credit to the original author(s) and the source, provide a link to the Creative Commons licence, and indicate if changes were made. The images or other third party material in this article are included in the article's Creative Commons licence, unless indicated otherwise in a credit line to the material. If material is not included in the article's Creative Commons licence and your intended use is not permitted by statutory regulation or exceeds the permitted use, you will need to obtain permission directly from the copyright holder. To view a copy of this licence, visit http://creativecommons.org/licenses/by/4.0/.

\section{References}

1. Adhikari SP et al (2020) Epidemiology, causes, clinical manifestation and diagnosis, prevention and control of coronavirus disease (COVID-19) during the early outbreak period: a scoping review. Infect Dis Poverty 9(1):29

2. Wang $Y$ et al (2020) Unique epidemiological and clinical features of the emerging 2019 novel coronavirus pneumonia (COVID-19) 
implicate special control measures. J Med Virol. https://doi.org/ 10.1002/jmv. 25748

3. Poissy J et al (2020) Pulmonary embolism in patients with COVID-19: awareness of an increased prevalence. Circulation 142(2):184-186

4. Sardu C et al (2020) Hypertension, thrombosis, kidney failure, and diabetes: is COVID-19 an endothelial disease? A comprehensive evaluation of clinical and basic evidence. J Clin Med 9(5):1417

5. Mao L et al (2020) Neurologic manifestations of hospitalized patients with coronavirus disease 2019 in Wuhan, China. JAMA Neurol 77:683

6. Shi S et al (2020) Association of cardiac injury with mortality in hospitalized patients with COVID-19 in Wuhan, China. JAMA Cardiol 5:802

7. Ackermann M et al (2020) Pulmonary vascular endothelialitis, thrombosis, and angiogenesis in Covid-19. N Engl J Med 383(2):120-128

8. Winkler ES et al (2020) SARS-CoV-2 infection of human ACE2transgenic mice causes severe lung inflammation and impaired function. Nat Immunol 21(11):1327-1335

9. Zhang $\mathrm{H}$ et al (2020) Expression of the SARS-CoV-2 ACE2 receptor in the human airway epithelium. Am J Respir Crit Care Med 202(2):219-229

10. Hamming I et al (2004) Tissue distribution of ACE2 protein, the functional receptor for SARS coronavirus. A first step in understanding SARS pathogenesis. J Pathol 203(2):631-637

11. Hoffmann M et al (2020) The novel coronavirus 2019 (2019$\mathrm{nCoV}$ ) uses the SARS-coronavirus receptor ACE2 and the cellular protease TMPRSS2 for entry into target cells. bioRxiv 60:1136

12. Delorey TM et al (2021) COVID-19 tissue atlases reveal SARSCoV-2 pathology and cellular targets. Nature 595(7865):107-113

13. Herold $T$ et al (2020) Elevated levels of IL-6 and CRP predict the need for mechanical ventilation in COVID-19. J Allergy Clin Immunol 146(1):128-136

14. Del Valle DM et al (2020) An inflammatory cytokine signature predicts COVID-19 severity and survival. Nat Med 26(10):1636-1643

15. Ziegler CGK et al (2020) SARS-CoV-2 receptor ACE2 is an interferon-stimulated gene in human airway epithelial cells and is detected in specific cell subsets across tissues. Cell 181(5):1016-1035

16. Johnson MC et al (2020) Optimized pseudotyping conditions for the SARS-COV-2 spike glycoprotein. J Virol. https://doi.org/10. 1128/JVI.01062-20

17. Onabajo $\mathrm{OO}$ et al (2020) Interferons and viruses induce a novel truncated ACE2 isoform and not the full-length SARS-CoV-2 receptor. Nat Genet 52(12):1283-1293

18. Ng KW et al (2020) Tissue-specific and interferon-inducible expression of nonfunctional ACE2 through endogenous retroelement co-option. Nat Genet 52(12):1294-1302

19. Blume $\mathrm{C}$ et al (2021) A novel ACE2 isoform is expressed in human respiratory epithelia and is upregulated in response to interferons and RNA respiratory virus infection. Nat Genet 53(2):205-214

20. Huang J et al (2020) SARS-CoV-2 infection of pluripotent stem cell-derived human lung alveolar type 2 cells elicits a rapid epithelial-intrinsic inflammatory response. Cell Stem Cell 27(6):962-973

21. Jacob ST et al (2020) Ebola virus disease. Nat Rev Dis Primers 6(1):13

22. Davey RA et al (2017) Mechanisms of filovirus entry. Curr Top Microbiol Immunol 411:323-352

23. Towner JS et al (2005) Generation of eGFP expressing recombinant Zaire ebolavirus for analysis of early pathogenesis events and high-throughput antiviral drug screening. Virology 332(1):20-27

24. O'Donnell KL, Marzi A (2021) Immunotherapeutics for ebola virus disease: hope on the horizon. Biologics 15:79-86
25. Dyall J et al (2017) Interferon-beta and interferon-gamma are weak inhibitors of ebola virus in cell-based assays. J Infect Dis 215(9):1416-1420

26. McCracken IR et al (2021) Lack of evidence of angiotensin-converting enzyme 2 expression and replicative infection by SARSCoV-2 in human endothelial cells. Circulation 143(8):865-868

27. Zhao Y et al (2020) Single-cell RNA expression profiling of ACE2, the receptor of SARS-CoV-2. Am J Respir Crit Care Med 202(5):756-759

28. Ziegler CGK et al (2021) Impaired local intrinsic immunity to SARS-CoV-2 infection in severe COVID-19. Cell 184(18):4713-4733

29. Zhou $Z$ et al (2020) Heightened innate immune responses in the respiratory tract of COVID-19 patients. Cell Host Microbe 27(6):883-890

30. Lee JS et al (2020) Immunophenotyping of COVID-19 and influenza highlights the role of type I interferons in development of severe COVID-19. Sci Immunol. https://doi.org/10.1126/sciim munol.abd 1554

31. Hadjadj J et al (2020) Impaired type I interferon activity and inflammatory responses in severe COVID-19 patients. Science 369(6504):718-724

32. Blanco-Melo D et al (2020) Imbalanced host response to SARSCoV-2 drives development of COVID-19. Cell 181(5):1036-1045

33. Bastard $\mathrm{P}$ et al (2021) Autoantibodies neutralizing type I IFNs are present in $\sim 4 \%$ of uninfected individuals over 70 years old and account for $\sim 20 \%$ of COVID-19 deaths. Sci Immunol 6(62):eabl4340

34. McKechnie JL, Blish CA (2020) The innate immune system: fighting on the front lines or fanning the flames of COVID-19? Cell Host Microbe 27(6):863-869

35. Nile SH et al (2020) COVID-19: Pathogenesis, cytokine storm and therapeutic potential of interferons. Cytokine Growth Factor Rev 53:66-70

36. Park A, Iwasaki A (2020) Type I and type III interferons-induction, signaling, evasion, and application to combat COVID-19. Cell Host Microbe 27(6):870-878

37. Broggi A et al (2020) Type III interferons disrupt the lung epithelial barrier upon viral recognition. Science 369(6504):706-712

38. Wang $\mathrm{N}$ et al (2020) Retrospective multicenter cohort study shows early interferon therapy is associated with favorable clinical responses in COVID-19 patients. Cell Host Microbe 28(3):455-464

39. Sposito B et al (2021) The interferon landscape along the respiratory tract impacts the severity of COVID-19. Cell 184(19):4953-4968

40. Eppihimer MJ et al (2002) Expression and regulation of the PD-L1 immunoinhibitory molecule on microvascular endothelial cells. Microcirculation 9(2):133-145

41. Fosse JH et al (2021) Endothelial cells in emerging viral infections. Front Cardiovasc Med 8:619690

42. Li S et al (2020) SARS-CoV-2 triggers inflammatory responses and cell death through caspase-8 activation. Signal Transduct Target Ther 5(1):235

43. Varga $Z$ et al (2020) Endothelial cell infection and endotheliitis in COVID-19. Lancet 395(10234):1417-1418

44. Xie X et al (2020) An infectious cDNA Clone of SARS-CoV-2. Cell Host Microbe 27(5):841-848

Publisher's Note Springer Nature remains neutral with regard to jurisdictional claims in published maps and institutional affiliations. 